\title{
Biological, Medical and Nano Structured materials - NMR done Simply
}

\author{
J Beau W Webber* \\ Department of Nano science, Marlowe Innovation Centre, Marlowe Way, UK
}

*Corresponding author: J Beau W Webber, Department of Nano science, Marlowe Innovation Centre, Marlowe Way, UK.

Received Date: April 26, 2019

Published Date: April 30, 2019

\begin{abstract}
Nuclear Magnetic Resonance (NMR) is a powerful technique that has many applications and a variety of techniques. It is often forgotten how simply it can be applied, particularly for material analysis. New highly portable NMR instrumentation based on Field Programmable Gate Array (FPGAs) is making the use of NMR in the laboratory and in the field fast and easy to use for materials research and characterization for biological and medical applications. It is particularly appropriate when nano-pores are present.
\end{abstract}

Keywords: Nuclear magnetic resonance (NMR); NMR relaxation; NMR T1; NMR T2; Material science; Nanopores

\section{Introduction}

We have all see the amazing detailed 3D images of the insides of people, that the non-invasive technique NMR Imaging is bringing us. And increasingly, it is not just simple images but also images with parameterized information based on the two common NMR relaxation times: T2, the time for the atoms to "share" the spin information, and T1, the time for the spin information to be lost to the "lattice" - the rest of the sample [1]. But these imaging systems are highly complex. The chemist's probe into molecules and pull out chemical structure and are always looking for magnets with higher and higher frequencies - now into the GHz region for water as well as extremely high homogeneity [2]. But let us not forget where NMR started, in the material sciences laboratories, with simple relaxation measurements of small samples at a few $10 \mathrm{MHz}$. Is this still of any interest? Well it certainly should be.

\section{Discussion}

There are a whole range of samples, from food, to biological, to rubbers and polymers, to medical materials, where the physical properties are open to study by NMR. In many of these materials these properties change with temperature, age or exact composition, and changes in these physical properties usually means changes in the NMR properties, particularly T1 \& T2. NMR is particularly adept at studying the properties of liquids such as water (and ice) at interfaces, or in Nanopores, as in [3] (Figure 1).

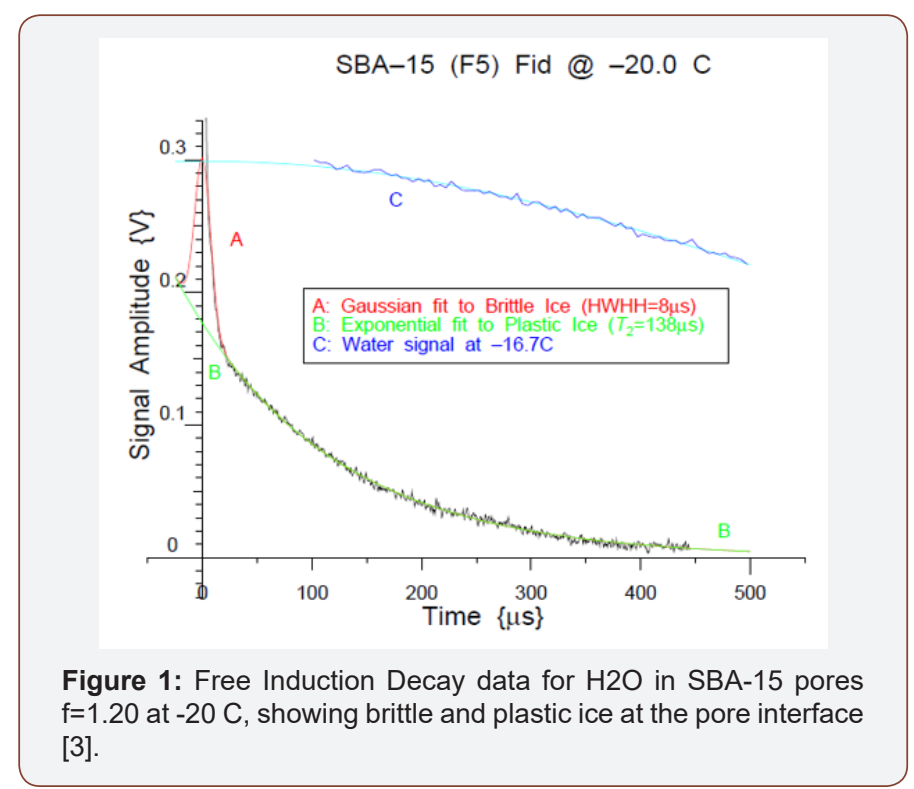

These studies can also throw light on the behavior of water at biological interfaces [4]. But you will say, even simple NMR instruments are still large and complex? Well no longer! Six components are required: a probe to hold the sample in the NMR coil; a magnet in which to put the probe and sample; a transmitter to create all the timing and pulse sequences to generate very short but powerful RF pulses (which are all contained inside the probe); 
a very sensitive and low-noise RF receiver, to capture and average the tiny NMR signals; and a console from which to control the experiment and analyses and display the results. This still sounds daunting, but two things have happened:

Firstly, magnetic materials have improved, and tiny but powerful permanent magnets suitable for NMR can be small enough to be hand-held, and can be taken to the sample being studied to measure properties such a aging in a racing car tire, or the maturity of "hung" meat. At its simplest the magnet can be just a cube of magnetic material. Slightly larger magnets but still smaller than a shoe-box, such as that shown in Figure 2, give results that are higher field/frequency, and have better homogeneity of field, so the results can be easier to interpret. (Figure 2).

Figure 2: Five of the components of a highly portable NMR relaxation spectrometer: Magnet, Probe, Receiver with Field Programmable Gate Array and Transmitter.

Secondly, Field Programmable Gate Arrays (FPGAs) are revolutionizing many instruments in the medical field and in the research laboratory, and NMR is now no exception. Figure 2 shows the basic components of a NMR instrument suitable for measuring physical properties A magnet - the one shown gives $24 \mathrm{MHz}$ for water; a probe - this sometimes needs to be customised for a particular experiment, and temperature control can be added; an NMR receiver (shown on the left) and an NMR transmitter (shown on the right), now both about the size of credit-cards (Figure 3).

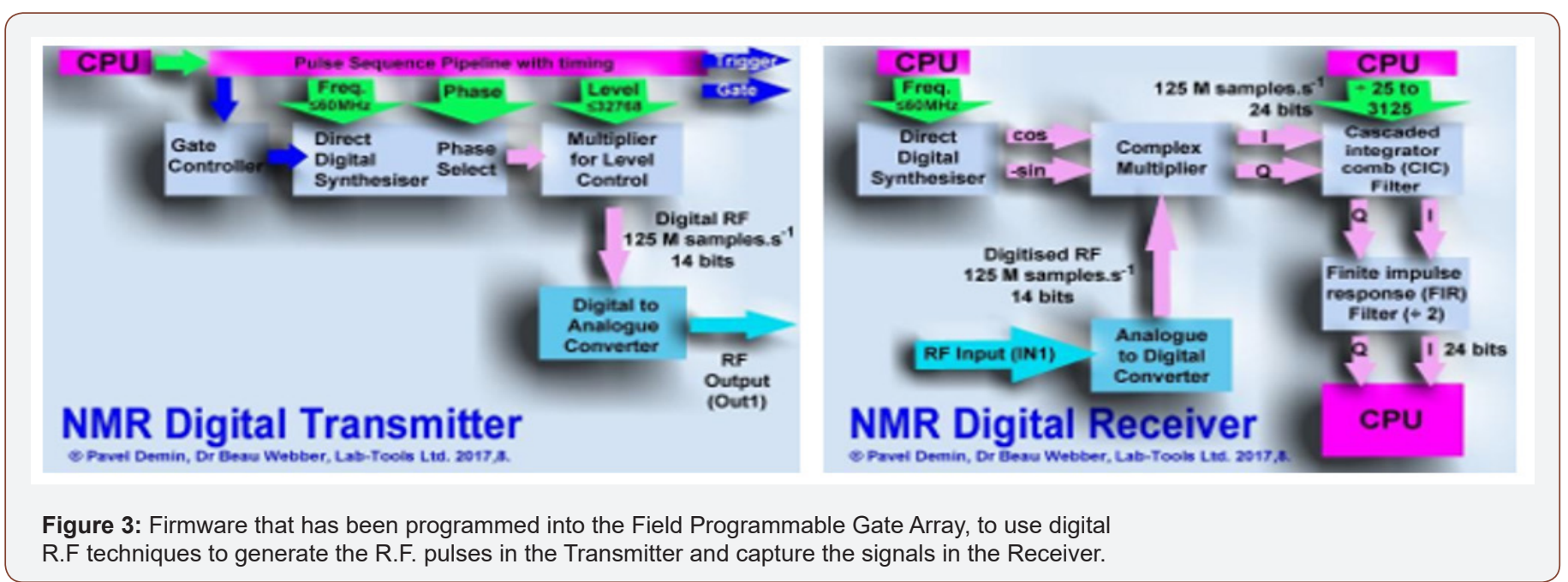

The digital sections of both the NMR receiver and transmitter are in an FPGA in the little credit-card sized black box under the receiver - a Red Pitaya - a Linux computer on an FPGA [5] with firmware and software that has been customized for NMR. A standard desktop or laptop is also needed for instrument control and filing the data, see (Figure 4). 


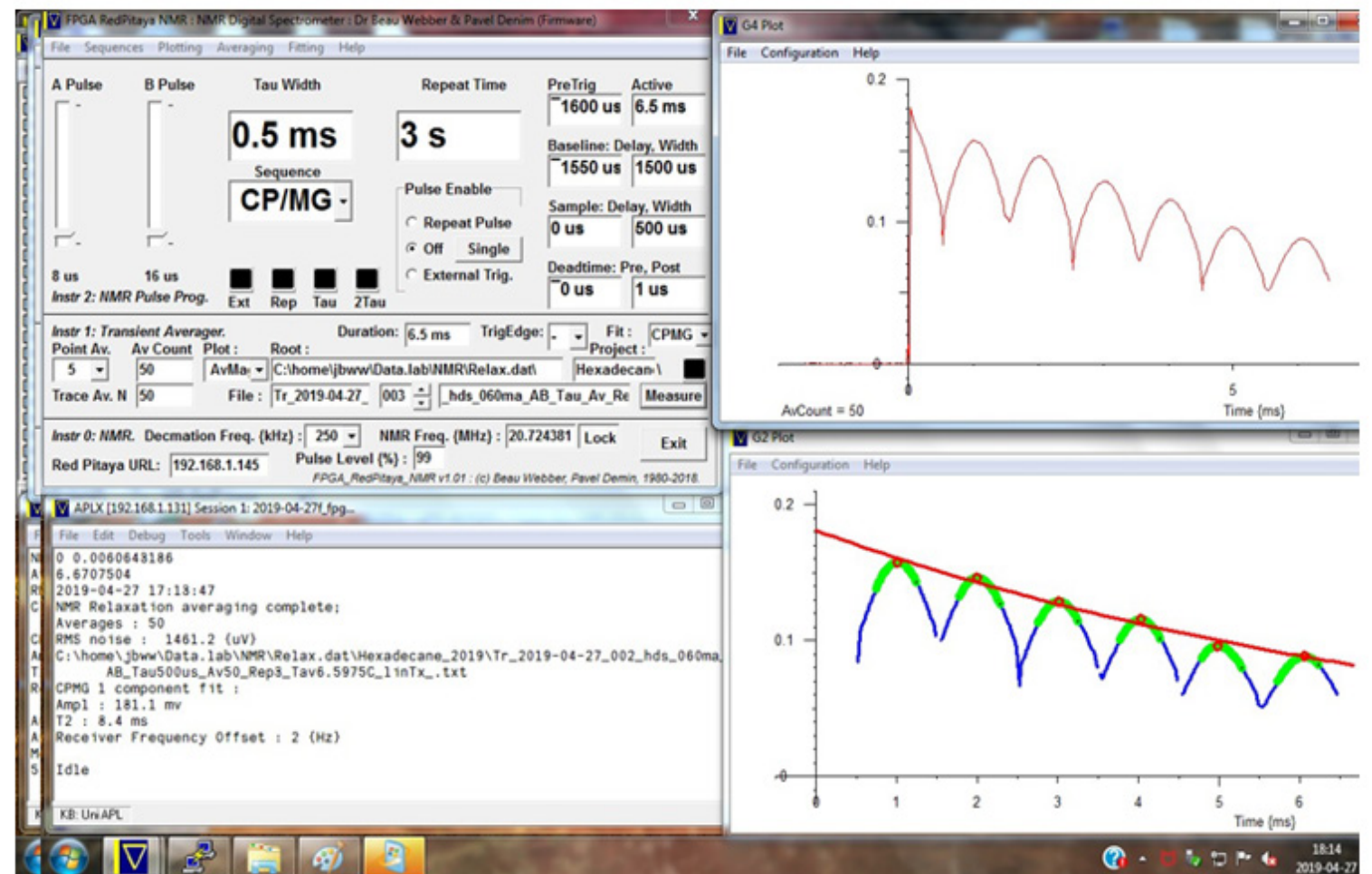

Figure 4: An example instrumental software front panel (Graphical User Interface) showing an averaged capture of a train of NMR echoes, to measure the T2 relaxation time of liquid hexadecane in the pores of a porous silica. The echo peaks are each polynomial fitted and then the decay of the peak maximum is exponentially fitted, with the results being reported during the measurement and logged to file.

These are the first offerings in the latest versions of digital NMR Relaxation Spectrometers [6] produced by a UK based NMR/ Nano-science company Lab- Tools Ltd. A new pore-size measuring instrument based on this NMR Spectrometer will also be released soon, covering a pore range from less than $1 \mathrm{~nm}$ to over $1 \mu \mathrm{m}$. Related designs have been in use in the laboratory making contract measurements for decades, using both NMR relaxation [7] and a technique called NMR Cryoporometry $[8,9]$.

\section{Conclusion}

A brief introduction has been given to the application of Nuclear Magnetic Resonance to materials research and characterization, suitable for biological and medical applications, using the latest highly compact Field Programmable Gate Array based NMR Relaxation Spectrometers. The lightness and compactness of these instruments make them ideal for use in an already busy laboratory, or for on-the-spot characterization in the field.

\section{Acknowledgement}

Firmware in Red Pitaya by Pavel Demin: https://github.com/ pavel-demin/red-pitaya-notes/

\section{Conflict of Interest}

No conflict of interest.

\section{References}

1. https://en.wikipedia.org/wiki/Relaxation_(NMR).

2. https://en.wikipedia.org/wiki/Nuclear_magnetic_resonance spectroscopy.

3. J Beau W Webber, John H Strange, Philip A Bland, Ross Anderson and Bahman Tohidi (2008) Dynamics at Surfaces: Probing the dynamics of polar and a-polar liquids at silica and vapour surfaces. AIP Conference Proceedings Series 1081(1): 51. Martin D Hürlimann, Yi Qiao Song, Paola Fantazzini and Villiam Bortolotti (2008) MAGNETIC RESONANCE IN POROUS MEDIA Proceedings of the 9th International Bologna Conference on Magnetic Resonance in Porous Media (MRPM9). AIP Conference Proceedings Series 1081.

4. JC Dore, MC Bellissent Funel, A Burian, HP Castricum, J Jelassi, et al. (2009) Structural studies of water in a confined hydrophobic environment. Journal of Physics: Conference Series 177(1).

5. https://www.redpitaya.com/.

6. http://www.lab-tools.com/instrumentation/.

7. https://petrowiki.org/Porosity_determination_with_NMR_logging.

8. https://en.wikipedia.org/wiki/Thermoporometry_and_ cryoporometry\#Nuclear_magnetic_resonance_cr yoporometry.

9. J Mitchell, J Beau W Webber, JH Strange (2008) Nuclear Magnetic Resonance Cryoporometry. Physics Reports 461(1): 1-36. 